\title{
versants
}

\section{Kramer avant Kramer : les récits des années 1980}

\author{
Daniel MaggetTI \\ Université de Lausanne
}

\begin{abstract}
La carrière littéraire de Pascale Kramer a pris son essor au milieu des années I990, au moment où l'écrivaine, établie à Paris, a commencé à y publier à un rythme régulier des romans dont le premier déjà, Manu (1995), paru chez Calmann-Lévy, est fortement remarqué ; il connaît un succès particulier en Suisse, où il obtient le prix Michel-Dentan. Cet article se propose de revenir sur les premiers pas en littérature de Pascale Kramer, en présentant les deux récits édités à Lausanne qui l'ont révélée au début des années 1980. En retraçant leur réception et en tentant de cerner leurs spécificités, mais en demeurant prudent vis-à-vis de toute perspective téléologique, on sera attentif aux éléments qui peuvent paraître annoncer des développements des œuvres de la maturité de l'auteure.
\end{abstract}

Keywords : Pascale Kramer, société, famille, amour, nature, réception

En avril 1982, quelques mois après son vingtième anniversaire fêté en décembre 198I, Pascale Kramer fait son entrée en littérature. Son premier roman, Variations sur une même scène, est un des quatre titres avec lesquels les Éditions de l'Aire, à Lausanne, lancent une nouvelle collection, baptisée "Le coup de dés » et réservée à des auteurs n'ayant pas encore publié. La nouveauté de l'initiative prise par Michel Moret, le directeur de la maison, le fait que Pascale Kramer soit la benjamine des quatre femmes dont les textes ont été retenus, la singularité de son récit se conjuguent pour valoir à celui-ci une réception à la fois attentive et globalement positive. À plusieurs comptes rendus du livre s'ajoutent ainsi quelques entretiens désireux de cerner le phénomène, présenté comme exceptionnel, que représente cette très jeune romancière tout de noir vêtue, dont on apprend qu'elle vient d'abandonner l'université fréquentée pendant une année. Ces exercices de question-réponse mettent en lumière la fraîcheur d'une personne d'une « étrange timidité » et qui « semble sortir d'un roman "(Bernheim 1982) : en dépit d'une certaine crânerie et du détachement, voire du brin de cynisme qu'elle affiche, Pascale Kramer laisse souvent transparaître un manque d'expérience lié à son âge. Comme elle le racontera bien plus tard, la publication de Variations sur une même scène s'est faite pour ainsi dire sans coup férir :

À l'époque du gymnase, j'écrivais des petits textes courts, qui me valurent un prix à un concours d'été. Parmi les membres du jury, il y avait Jacques Chessex, mais aussi Raymond Denat, un professeur de français en fin de carrière, un homme un peu démodé, je crois pas très aimé [...]. 
Encouragée par mon prix, je m'étais lancée dans un roman dont Raymond Denat $^{1}$ suivait les progrès quasiment page à page. Nous nous retrouvions à la bibliothèque du gymnase dont il s'occupait entre ses cours. C'est grâce à sa patience que j'ai réussi à tenir la distance du roman (Kramer 20I0).

Une élève douée, un professeur dévoué, un concours littéraire providentiel : les débuts de Pascale Kramer lui laissent à peine le temps de se rendre compte qu'elle vient de changer de statut et que sa nouvelle qualité d'écrivain fait d'elle un personnage public dont le livre comme les propos sont pesés et jugés. D'aucuns relèvent ainsi que l'auteur de Variations sur une même scène "promène ses 20 ans et pas tout à fait six mois dans un petit monde qu'elle s'est fait à sa mesure », et que "son livre porte le regard qu'on jette à cet âge sur les gens et les choses » (Radio TV-Je vois tout 1982). Une chroniqueuse engagée, Christiane Mathys-Reymond, paraît s'étonner de l'absence de positionnement d'une jeune fille proclamant qu'elle n'est "pas particulièrement féministe », parce que « cela [l']ennuie de suivre une conduite stricte», et qu'elle veut " pouvoir [s']emballer pour un macho sans remords féministes" (Mathys-Reymond I982 : I2). La palme de la rosserie revient cependant à Anne Zirilli, dont l'article intitulé «Les paresses de Pascale » s'apparente à une exécution teintée de condescendance quelque peu méprisante. Aux yeux de la journaliste, « comme son personnage, Pascale Kramer cherche à faire le vide autour d'elle. Élève la paresse au rang des qualités charmantes. Préfère le refuge des rêveries au monde trop sérieux des adultes " (Zirilli $1982: 55$ ). À preuve de ce qui constitue à ses yeux une immaturité patente, Anne Zirilli souligne le sentiment de déception ressenti par Pascale Kramer au gymnase, son abandon des études académiques, et insiste sur des confidences qu'elle lui a soutirées en abusant de sa naïveté :

Les voyages la rebutent, avec leurs risques de trains manqués et de bus pris à rebrousse-poil. Le travail aussi, avec ses exigences réalistes. Sauf s'il permet de jouer avec les mots, que ce soit en publicité, dans le journalisme ou dans l'édition... trois secteurs dans lesquels Pascale Kramer s'active avec un enthousiasme novice.

Sa carrière littéraire a commencé tôt : à 9 ans, une histoire de chats, à $\mathrm{I} 7$ ans une série de « Portraits », qui lui valent le Prix du Gymnase de la Cité et une petite gloire de quartier. Seul membre du jury, Jacques Chessex lui met dix sur dix (Zirilli I982: 55).

L'affaire paraît entendue, souffle-t-on au lecteur : le jeune prodige est en réalité une oie blanche ou une petite fille gâtée, propulsée au rang d'auteur

I Raymond Denat est un des deux dédicataires de Variations sur une même scène, l'autre étant Sylvie Colbois, amie et condisciple de Pascale Kramer au Gymnase de la Cité à Lausanne. 
grâce au bon vouloir, ou au caprice, d'un écrivain à fort capital symbolique, Jacques Chessex, qui au début des années I980 est réputé pour faire la pluie et le beau temps dans le landerneau des lettres romandes. Cette présentation narquoise a obligé Pascale Kramer à prendre conscience des aléas d'une activité dont elle n'avait pas eu le temps de mesurer les retombées : on devine son sentiment - et sa déception - à la lecture d'une lettre de lecteur qu'elle envoie le I6 avril I982 au périodique dans lequel a paru l'article malveillant.

La réception du récit en tant que tel, indépendamment des commentaires qu'inspirent l'âge ou la personnalité de son auteur, n'en demeure pas moins globalement positive. Certes Yves Bridel, dans une brève chronique portant sur les quatre premiers «Coups de dés », dit ne pas avoir été convaincu par Variations sur une même scène, tout en concédant qu'elles sont « beaucoup plus classiques et bien faites » que d'autres titres de la collection (Bridel 1982). Gilbert Salem, en revanche, admire que Pascale Kramer ait su éviter les pièges dans lesquels tombent souvent les débutants, en fuyant toute tentation autobiographique au profit d'une vraie mise en forme fictionnelle:

Il s'agit d'un roman de fiction, une histoire imaginée, construite avec adresse, intelligence et sensualité. L'approche des personnages est originale, lucide, mais non sans une certaine chaleur. La phrase est structurée avec finesse et componction, quelquefois au point d'étouffer les émotions. Mais le goût de l'aventure des mots et l'audace des néologismes la sauvent, laissant fuser un admirable bagou (Salem I982).

Les particularités de l'écriture kramerienne, qui repose à la fois sur une structuration originale de la phrase, sur le recours à un lexique parfois forgé de toutes pièces et sur un goût certain pour les catégories non concrètes, semblent frapper davantage que les éléments de l'intrigue échafaudée par la jeune écrivaine. À l'approche d'une émission dans le cadre de "La Librairie des ondes », un article de Radio TV - Je vois tout définit ainsi le roman comme " une suite de notations où les descriptions utilisent des termes tout d'abstraction, où les portraits des personnages sont définis par des appréciations plus morales que physiques », d'où " pourtant il se dégage beaucoup de présence dans les personnages, pas mal de réalisme dans les décors ». Et le chroniqueur de conclure :

Bref, ce livre est un roman construit selon les règles du genre, mais d'une étonnante sécheresse de forme, et qui se réclame davantage du classicisme que de la grande tradition romanesque telle que nous l'avons héritée du siècle dernier (Radio TV-Je vois tout 1982 ).

La suggestion d'un rattachement à la tradition classique davantage qu'à la pratique réaliste et naturaliste du roman est sans doute suscitée par le fait 
que le premier roman de Pascale Kramer est aux antipodes de la mise en récit balzacienne. Les figures qui traversent Variations sur une même scène n'ont pas de nom de famille, parfois même pas de prénom, comme le vieil homme qui héberge gracieusement Jacques, le personnage principal. Réduite à une épure, l'histoire racontée est dépourvue de tout arrière-plan socio-politique ; elle s'ingénie à explorer à travers quelques épisodes les hésitations, les errances et les palinodies de Jacques, velléitaire face à de vagues projets artistiques, superficiel dans les deux relations amoureuses fatalement avortées qu'il noue, avec Carole, une jeune fille de son âge, et avec Béatrice, une femme plus âgée. Le récit aboutit ainsi à dessiner les contours de sa personnalité morale, de sa "nature ", dans une perspective qui est bien celle de la «peinture des âmes » de l'âge classique. C'est à ce constat que parvient Claire Jaquier dans le compte rendu qu'elle donne dans le "Samedi littéraire » du 22 mai 1982 :

Pascale Kramer nous propose [...] un roman où se découvre la superficialité des êtres : les personnages cherchent, sans volonté ni conviction, la séduction, l'approbation, le prestige, la gloire ou simplement une image d'euxmêmes. Leurs amours les lient à peine, leurs tentatives artistiques ne sont que le reflet d'un désir de gloire, future et improbable. Jacques, le personnage central, apparaît vidé de toute profondeur, au bord de la disparition [...]. Pascale Kramer cerne d'une plume habile ce thème si fuyant de l'êtreen-miroir (Jaquier 1982).

Bien que souvent concis, les échos critiques de Variations sur une même scène au moment de sa parution ne manquent pas de pertinence. Ils soulignent comment Pascale Kramer déjoue les attentes de lecture en s'en tenant à une histoire minimale en termes d'action et de péripéties, et en se focalisant uniquement sur la personnalité peu consistante d'un protagoniste dont l'indolence et la paresse peuvent être lues comme le symptôme du mal de toute une génération en manque d'engagement et de motivation. Les jeunes personnages n'envisagent pas de reconduire les modèles de leurs aînés, comme le montre la révolte de Jacques vis-à-vis de sa mère, furtivement croquée comme la quintessence d'un système bourgeois bien-pensant et sclérosé. N'embrassant aucune voie, Jacques ne prend aucun risque, mais se condamne volontairement à une sorte de stagnation dans laquelle il semble se complaire. Celle-ci affecte aussi ses amours : l'impasse socio-professionnelle se double fatalement d'un échec sur le versant de la vie intime. Centré sur Jacques, le roman glisse plus rapidement sur les personnages féminins, qui apparaissent cependant tiraillés entre la soumission à des injonctions de rôle traditionnelles, et un désir d'indépendance et d'auto-détermination. 
En privilégiant la restitution éclatée des traits de caractère qui, rassemblés, donnent forme au portrait psychologique de Jacques, mais aussi en préservant une part de mystère autour d'autres silhouettes, en particulier celles de Béatrice et du vieil homme, Pascale Kramer révèle dans Variations sur une même scène la perspicacité de son regard et l'habileté de son approche des êtres. Par touches progressives, le récit met notamment en évidence l'ambiguïté du personnage de Jacques, dont la veulerie n'efface pas le pouvoir de fascination : en évitant d'asséner des jugements de valeur, l'instance narrative fait percevoir la complexité de la relation à autrui, la difficulté, voire l'impossibilité, d'appliquer des catégories définitives qui permettraient de classer des comportements ou des caractères en se faisant d'eux une opinion arrêtée une fois pour toutes. Cette opacité de la nature humaine, mouvante, impénétrable, souvent imprévisible, est un trait qui émergera dans plusieurs des fictions postérieures de Pascale Kramer ; avec Jacques, elle aura mis en place dès son entrée en écriture une sorte de prototype des personnages qui l'intéressent. Variations sur une même scène a ainsi offert à la jeune auteure la possibilité d'expérimenter les potentialités d'un style en train de tester ses ressources et ses trouvailles, et de dévoiler une approche des figures romanesques et une vision du monde qu'elle n'aura de cesse de souligner dans la suite de son œuvre.

L'aspect exploratoire est plus frappant encore dans Terres fécondes (1985), que Pascale Kramer fait paraître, toujours à l'enseigne de l'Aire, au printemps I985. Il s'agit bien, comme annoncé dès I982, d'« un conte se passant dans un pays imaginaire " (Radio TV-Je vois tout 1982) : il met en scène un jeune garçon, Bruno, évoluant entre le village paysan d'où il est issu et le château « inutilement immense » du seigneur Mormorain, au fil d'un parcours initiatique retracé dans une tonalité qui oscille entre le fantastique et le merveilleux. Épousant le cycle des saisons, le récit «imagin[e] une évolution accélérée » (S.M. 1985) et raconte l'inexorable défaite du monde des hommes face à une nature impossible à contenir. La vigueur luxuriante des éléments, et tout particulièrement de la végétation, aura raison des structures mises en place par la société pour asseoir la légitimité de son existence et pour accroître son autorité : aux deux bouts de la chaîne de l'existence, Bruno comme Mormorain font l'apprentissage de la vanité des espoirs individuels et des croyances collectives. Dans un univers où tout se délite et qui paraît condamné au retour à l'indistinction de la boue originelle, les droits des forces fertiles qui imposent leur loi efface tout autre repère. Entre la fable et l'allégorie, ce petit livre se détache de toute vraisemblance réaliste pour proposer au lecteur des questionnements aux ambitions dignes de celles d'un conte philosophique, ainsi que le signale un compte rendu : 
Ce fantasme [celui d'une vie modelée par le végétal] s'accompagne d'un désarroi dont les personnages reflètent les divers aspects et leurs conflits. Faut-il résister ou se plier quand la nature est victorieuse ? Que reste-t-il de l'homme? Assistons-nous à une chute, désagrégation sans issue, ou à une nouvelle naissance? Les deux, bien sûr, si comme l'adolescent Bruno nous découvrons que c'est l'adaptation et le refus de l'immobilisme qui fondent notre humanité (Reymond 1985).

D'aucuns se laissent prendre à un « fil secret et étrange " qui « se fraie un chemin inattendu dans ce labyrinthe d'immobilité anxieuse, où couvent des sentiments exacerbés » (S.M. 1985); pour d'autres, « les personnages et plus encore le message [...] s'estompent »(B[ridel] I985). Tous s'accordent pour reconnaître à Terres fécondes deux qualités majeures. Tout d'abord, la capacité à " créer un climat poétique, à rendre sensible la fécondité oppressante de la nature, à évoquer les sensations et les sentiments de Bruno » : le livre est ainsi assimilé à « un poème mythique ou symbolique » (B[ridel] 1985). Ensuite, l'originalité du style ; cette langue qui « accumule redondances, excroissances et volutes servies par un vocabulaire riche et imagé » (Reymond 1985) est généralement jugée en parfait accord avec l'atmosphère du récit ${ }^{2}$ : «l'écriture de Pascale Kramer envoûte par la succulence de son vocabulaire, la préciosité de ses méandres, l'épaisseur touffue et drue de ses images» (S.M. 1985).

Terres fécondes paraît cependant a posteriori comme un moment quelque peu troublant sur le parcours de Pascale Kramer. Le choix générique du conte est-il dû au désir d'explorer d'autres formes que celle du récit faisant référence à des réalités contemporaines ? S'il se révèle être un véritable laboratoire d'écriture, dans la mesure où l'auteur y essaie et y déploie les draperies de son incomparable phrasé, le récit tient par certains côtés de l'exercice de style : les sentiments des personnages sont certes bien saisis, mais ces figures n'ont pas l'ancrage, fût-il minimal, qui les rendrait crédibles en les inscrivant dans un contexte plus familier à l'écrivain. Encore peu expérimentée, Pascale Kramer a peut-être manqué, après son coup d'essai, d'un accompagnement comparable à celui que lui avait garanti Raymond Denat pour Variations sur une même scène. Ses deux premiers livres lui ont probablement permis de mieux percevoir dans quel sens il lui faudrait creuser pour que son talent s'épanouisse pleinement. Elle ne cessera de le faire pendant les dix ans qui séparent la parution de Terres fécondes (1985) de celle de Manu (1995) - en travaillant à divers projets qui ne verront pas le jour (les a-t-elle

2 Même s'il se montre plus sévère, Michel Vuillomenet, après avoir trouvé que le texte dans un premier temps « reste très abstrait, artificiel, comme gêné aux entournures par l'ampleur voulue du propos », juge lui aussi que " assez vite l'écriture s'assouplit dans le récit de l'action, saisit mieux, avec plus de naturel et de pouvoir évocateur, un jeu de la vie et de la mort laissant comme exténué et pantois cet adolescent qui a poussé trop vite» (Vuillomenet 1985). 
conservées dans ses archives ?) et dont seules des bribes sont connues (on songe notamment à un extrait publié dans la revue lausannoise Écriture en I988). Sans doute lui a-t-il fallu ce temps de sédimentation, et quelques tentatives inabouties, pour parvenir à maîtriser la profusion de son écriture, non pour la brider, mais pour en orienter plus efficacement les effets. Relire les deux premiers livres de Pascale Kramer, c'est ainsi entrer en contact avec les virtualités d'une œuvre qui tâtonne et qui se cherche, mais qu'un vent créateur ébouriffe déjà.

\section{Bibliographie}

Anonyme, «Pascale Kramer : 20 ans et un roman!", Radio TV-Je vois tout, 27/05/1982.

Bernheim, Patricia, "Pascale, écrivain », Le Matin / Tribune de Lausanne, 5/07/1982.

B[ridel], Y[ves], «Le "Coup de dés" de l'Aire. Un poker d'as ? », 24 heures, 20/07/1982.

Bridel, Yves, «Terres fécondes de Pascale Kramer», 24 heures, 26/06/1985.

Jaquier, Claire, "Jamais un coup de dés...», "Samedi littéraire », Gazette de Lausanne et Journal de Genève, 22/05/1982.

Kramer, Pascale, Variations sur une même scène, Lausanne, Éditions de l'Aire, 1982.

—. «Un abus de confiance », rubrique « Courrier» de L'Hebdo, I6/04/1982, p. 62.

-. Terres fécondes, Lausanne, Éditions de l'Aire, 1985.

—. « La dédicace à Véréna ", Écriture 3I, automne I988, pp. 2II-2I3.

—.Manu, Paris, Calmann-Lévy, 1995.

-. Réponse à la laudatio [au prix Rambert 20Io], en ligne: www.prix-rambert.ch (consulté le 30/or/2020).

Mathys-Reymond, Christiane, "Pascale Kramer : Variations sur une même scène », entretien, Femmes suisses, 70/II, novembre 1982, p. I2.

Reymond, Félicie, « Lettres romandes. Le règne du végétal », « Samedi littéraire », Gazette de Lausanne et Journal de Genève, I7/08/1985.

Salem, Gérard, «Des premiers livres pleins de souffle», 24 heures, 13/04/1982.

S., M., "Terres fécondes, de Pascale Kramer ", Journal et feuille d'avis de Vevey-Riviera, 26/06/1985.

Vuillomenet, Michel, «Terres fécondes, de Pascale Kramer : une allégorie en progrès... », L'Est vaudois, 22/11/1985.

Zirilli, Anne, «Les paresses de Pascale », L'Hebdo, 8/04/1982, p. 55. 
